\title{
Variable Rate Adaptive Modulation (VRAM) for Introducing Small-World Model into WSNs
}

\author{
Waqar Asif *†, Hassaan Khaliq Qureshi* and Muttukrishnan Rajarajan ${ }^{\dagger}$ \\ *National University of Sciences \& Technology (NUST), Islamabad, Pakistan. \\ Email: $\{09$ mscsewasif,hassaan.khaliq $\}$ seecs.edu.pk \\ ${ }^{\dagger}$ School of Engineering and Mathematical Sciences, City University, London, UK. \\ Email: \{waqar.asif.1,r.muttukrishnan\}@ city.ac.uk
}

\begin{abstract}
Data communication has a strong impact on the design of a Wireless Sensor Network (WSN), since the data transmission energy cost is typically higher than the data processing cost. In order to reduce the data transmission cost, small world phenomenon is introduced into WSNs. Networks that do not have the small world structure can be converted to achieve a small world property by the addition of few extra links. The problem is that most large scale WSNs are inherently unstructured and a node has no precise information of the overall model of the network and thus has to rely on the knowledge of its neighbor. For this reason, in most unstructured networks, information is propagated using gossiping. In this paper, we exploit this information propagation mechanism and use Neighbor Avoiding Walk (NAW), where the information is propagated to node that has not been visited previously and which is not the neighbor of a previously visited node. Using this, a novel approach is presented, in which nodes with highest betweenness centrality form a long distance relay path by using a lower order modulation scheme and therefore resulting in a relatively reduced data rate, but maintaining the same bit error rate. Our empirical and analytical evaluations demonstrate that this leads to a significant reduction in average path length and an increase in node degree.
\end{abstract}

\section{INTRODUCTION}

The development of compact and intelligent nodes equipped with self-configuring and communication capabilities have promoted the emergence of Wireless Sensor Networks (WSN). WSNs contain nodes that cooperatively establish a network, to fulfill the needs of an infrastructure like entity for implementation of control functions. Each node in the network acts as a peer to its immediate neighbor and message routing relay node for its non-neighbor. These relayed messages cost in terms of reduced reliability [1]. They enable intermediate malicious nodes to alter relayed data in their own interest. In order to reduce this dependence and route messages quickly, small-world phenomenon is introduced into WSNs.

This small-world phenomenon was initially studied by Milgram [2] in context of social networks, who observed that the average separation between a source and a destination lies between five and six, and is subsequently termed as the "six degree of separation". Using this phenomenon Watts and Strogatz in [3] published the first small world model that used a single parameter to interpolate between a random graph and a lattice. They show that by randomly rewiring edges, of a regular graph, to form a small number of long range links while keeping the diameter in proportion to the network, the network can be reduced into a small-world network with small Average Path Length (APL) and large Clustering Coefficient
(CC). APL is defined as the mean of the shortest path lengths between all pair of vertices and it represents the closeness and consequently, how quickly information transfer takes place in a network [4].

Random edge addition can decrease the APL of a network, but such a reduction should be performed while keeping in consideration the compatibility and characteristics of the network, so that the properties of the network are not at stake. For instance, in WSNs it is inappropriate to enforce a mechanism that allows randomly selected nodes to be neighbor, and subsequently increasing their transmission radius to extend over the entire network, thus depleting the node energy instantly and making the network non-functional. A few attempts have been made in the past to identify different strategies for creating short-cut paths in WSNs. The designs proposed in existing literature require addition of extra infrastructure, such as high capacity nodes, additional radios and wires [1][5][6]. Therefore, the approaches require corresponding infrastructure and are limited to specific scenarios.

In this paper, we take a different perspective by focusing on exploiting the available hardware and increasing the transmission radius of a wireless device by using variable modulation scheme. Limited nodes, having the highest betweenness centrality are selected to form long range short-cut paths. The betweenness centrality of a node is calculated iteratively using Neighbor Avoiding Walk (NAW). In NAW, a node walks through a node, which has not been previously visited and is not a neighbor of a previously visited node and therefore providing better convergence time. Our analysis demonstrates that the proposed approach provides considerable reduction in APL.

The rest of the paper is as follows: Section II explains the related work, Section III elaborates the proposed algorithm, Section IV highlights the Simulation Results and Conclusion is presented in Section V.

\section{RELATED WORK}

In this section, some techniques are presented, which introduce the small world property into WSN's.

In [3], Watt and Strogatz proposed a model to construct a small world network out of a regular graph whose topology is of a low density regular lattice. A regular graph is rewired using the probability $p$, an edge is reconnected to a randomly chosen vertex, while avoiding duplicate edge formation. In the next phase, considering the probability $p$, the process is 
repeated for the edges that connect the vertex to the second nearest neighbor. This process continues circulating around the ring, unless each edge of the original lattice is considered once. Watt and Strogatz conclude that by using this model and rewiring only $1 \%$ of the edges and APL reduction of $80 \%$ can be achieved. Despite having such an advantage, this model is in-appropriate for use in WSNs. Implementing such a model would result in selecting random nodes in the network to become neighbors, forcing the nodes to extend their transmission radius over the complete network.

Considering the transmission radius limitation and spatial dependence of WSNs, Sharma and Mazumdar [5] used wired links to create shortcut paths in WSNs. These wired links have wireless transceivers attached at both ends to make it replenish their energy, as there is no energy constraint at the wire links. They show that by adding few wired-links in a WSN, hop count for multi-path routing can be reduced, resulting in reduced energy dissipation. A node intending to relay data to a far-away node shall forward it to the wiredlink, which shall transfer it to the other end, and by using wireless transceivers at both ends, forward data to its desired destination node. However, in many application scenarios like, node movement tracking, such a mechanism would not be able to facilitate because these wired-links once deployed will not be able to move along the movement of the wireless devices.

To solve this issue Chetan Kumar et al. in [6] equip gateway nodes with two radios, a short range and a long range. The long range radio is dedicatedly used for establishing a link between distant nodes. Addition of such links improves network performance by $25 \%$ and reduces the APL up to $43 \%$, but it is not feasible for mission critical sensor network applications where increasing the network lifetime is one of the prime focus. Use of extra hardware will deplete the node energy at a higher rate resulting in reduced network lifetime.

On the other hand, Eleni et al. in [7] have proposed a topology control based approach, intended to aid multi-hop communication in an intelligent and effective manner. They use directional antennas between nodes having the highest degree. A node having higher degree is more likely to be handling heavy traffic and is hence equipped with a shortcut link to reduce multi-hops for messages intended for distant nodes.

Likewise Abhik Banerjee et al. in [1] have reduced APL with the use of directional antennas along with a distributed self-organizing framework, which figures out highly connected nodes without by using node's traffic flow. They believe that nodes having higher traffic flow are better candidates to be connected for path reduction. Traffic flow in WSNs is bursty in nature [8] hence; the value of this parameter will change in a random order, resulting as a constant change in nodes selected to use directional antennas. Directional antennas have a limitation of reducing the covered angle for the increase in covered distance, hence if a node intends to cover a longer distance; it will have to direct its energy into a narrower beamwidth at the cost of losing connections with its neighbors. To overcome this deficiency we propose a new scheme.

To the best of our knowledge, no previous work has emphasized on increasing the transmission radius of a node by introducing variable modulation scheme. The proposed technique eliminates the deficiency faced in using directional antennas of reducing transmission angle for the increase of transmission distance. It uses neighbor avoiding walk mechanism for calculating betweenness centrality and selects a group of nodes that uses variable modulation technique for data transfer. Variable modulation scheme is employed to guarantee signal interpretation quality while adding to form long distance relay path without the use of any additional infrastructure.

\section{VARIABle RAte AdAPtive Modulation (VRAM)}

In this section, we discuss how shortcut paths can be added into the network, while using the available resources and maintaining the topological characteristics of the network.

\section{A. Motivation}

As WSNs are spatial graphs, it is possible to reduce the average minimum path length between nodes by simply adding shortcut paths. These paths can only benefit the network if deployed after properly considering the topological characteristics of the network. Since, WSN perform communication using broadcast mechanism, all neighbors receive the packet sent by a particular node. In order to reduce the average minimum path length between nodes, we need to add long range unicast links. The simplest way to add these links is by increasing the node transmission radius, but this radius is highly correlated with the energy consumption of a node. For a minimum communication radius of $R$, energy spent to transmit a signal is $R^{\alpha}$, where $\alpha$ is the path loss exponent [9]. Hence, an efficient mechanism is required that increases transmission radius while considering the dependence on energy consumption.

Our aim is to reduce the mean path length between two randomly selected nodes, while simultaneously maintaining the energy consumption of nodes, with the use of variable modulation. Variable modulation scheme enables nodes to cover a greater distance by just reducing the modulation scheme to a lower index, from $64 Q A M$ to $16 Q A M$. A higher order modulation scheme has a higher path loss exponent, increasing the bit error rate with increase in distance, hence by using a lower order modulation scheme $(16 Q A M)$ for a limited number of nodes, the overall data rate is not effected [10]. The selection procedure is explained in the next section.

\section{B. Centrality Indices Based On Neighbor Avoiding Walk}

Wireless Sensor Networks are conveniently described as graphs $G=(V, E)$, where $E$ represents the set of edges and $V$ represents the set of vertices. If we have a path from $a \in V$ to $b \in V$, beginning from $a$ and ending at $b$ then we can use $G_{d}(a, b)$ to denote the minimum distance between node $a$ and $b$. By definition $G_{d}(a, a)=0$ for every set of $a \in V$ and $G_{d}(a, b)=G_{d}(b, a)$ for any $a, b \in V$. Let $\sigma_{a, b}$ denote the number of shortest paths from $a \in V$ and $b \in V$, where by convention $\sigma_{a, a}=1$ for every set of $a \in V$. If $\sigma_{a, b}(z)$ denotes shortest path from $a$ to $b$ which passes through a node $z \in V$ than the betweenness centrality will be:

$$
B_{C}(z)=\sum_{(a, b, z) \in V} \frac{\sigma_{a b}(z)}{\sigma_{a b}}
$$

A high centrality score shows that a vertex can reach others on a relatively short path. For ease in understanding and to 
control the size of the network, the value of betweenness centrality is kept between zero and one. The size of a WSN makes the evaluation of betweenness centrality computationally expensive. Hence to compute the betweenness centrality we base ourselves on the Bellman criterion, according to which a vertex $z \in V$ lies on the shortest path between vertex $a, b \in V$, if and only if $G_{d}(a, b)=G_{d}(a, z)+G_{d}(z, b)$.

Given the shortest path and the pairwise distances, the pair dependency $\delta_{a b}(z)=\sigma_{a b}(z) / \sigma_{a b}$ of a pair $a, b \in V$ is on an intermediate vertex $z \in V$ [11]. Hence the ratio of the shortest path between $a$ and $b$ that $z$ lies on is given by:

$\sigma_{a b}(z)= \begin{cases}0 & \text { if } G_{d}(a, b)<G_{d}(a, z)+G_{d}(z, b) \\ \sigma_{a z} \cdot \sigma_{z b} & \text { otherwise }\end{cases}$

To obtain the betweenness centrality of a vertex $v$, we simply have to sum the pair-dependencies of all pairs of vertices, hence

$$
B_{C}(z)=\sum_{(a, b, z \in V)} \delta_{a b}(z)
$$

This shows that betweenness centrality can be determined in two steps:

1) Compute length of the shortest paths between all pair of vertex

2) Sum all pair dependencies

The complexity of determining the betweenness centrality is dominated by the second step, since $O\left(n^{2}\right)$ pair dependencies need to be summed for each vertex and the running time of the implementation is dominated by the time spent on matrix multiplication. It is clear that algebraic path counting computes more information than needed; hence traversal algorithms are used to exploit the scarcity of typical instances. Brandes et al. in [11] use both breath-first search (BFS) for un-weighted and Dijkstra's algorithm for weighted graphs. Considering the amount of nodes to be traversed for path counting, we use Neighbor Avoiding Walks (NAW) to reduce this overhead.

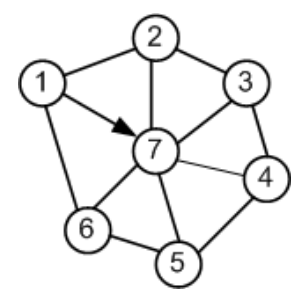

Fig. 1. Long Leg Capability.

In NAW, the walker starting from the sink node, does not visit any node previously visited up to a certain depth $n$ nor does it visit the neighbor of a previously visited vertex up to a distance $m$. Here as we consider the depth $n=1$ and distance $m=1$ then the neighbors not being covered by this rule will receive messages according to a random policy which is normally uniform.

Our policy of using NAW with $n=1$ and $m=1$ has two advantages: a long leg capability and an enhanced bridge crossing. Fig 1 highlights the first advantage: Suppose that a message that was located at vertex 1 at a previous time step and has now moved to vertex 7 . In case of a memory less system, where a node can visit any randomly selected node, it can be routed to all the neighbors of vertex 7 . Whereas according to NAW it can be routed to all the neighbors of vertex 7, except vertex 1 and neighbors of vertex 1 .

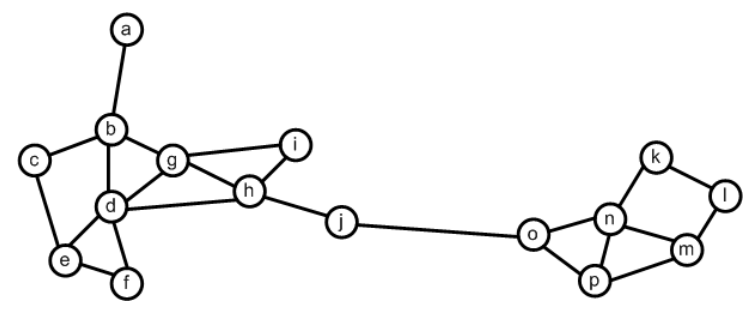

Fig. 2. Enhanced Bridge Crossing.

The second advantage of this mechanism is that, in a network shown in Fig 2, where the walk reaches from a wellconnected area to a node which represents the end of a bridge, then the favorite path of that walk is through the bridge, because by definition the node at the opposite end cannot be neighbor of the previous node.

The extra stiffness added in NAW by not visiting neighbors of the previously visited vertex results in an increased path walked by the walker due to the square root law of diffusion [12]. Once each node has been visited and betweenness centrality is calculated by all nodes in the network, then nodes having the highest betweenness centrality value form long distance links by using VRAM.

\section{Variable Rate Adaptive Modulation (VRAM)}

Mobile radio channels are prone to burst errors due to deep fades, even when the channel Signal to Noise Ratio (SNR) is kept high. This can be reduced by the use of variable transmission power or by varying the constellation size. In the former, with the increase in transmission power, the co-channel interference also increases [13]. This leads us to the notion of varying the constellation size under uniform transmission power, so that when a longer distance is to be covered, a lower order constellation is used and for a shorter distance a higher order constellation is used. This change in constellation size provides us with variable data rates, while maintaining a constant bit error rate (BER) [13]. Such an approach also helps avoid bit errors occurring in bursts, hence if we maintain a constant BER, and only change the modulation scheme than we can cover a longer transmission distance. In this discourse we intend to cover a longer distance by using conventional $16 Q A M$ and $64 Q A M$ modulation with square constellation as shown in Fig 3.

In this work, we assume that the interference from other nodes can be modeled as Gaussian noise and the effect of this interference can be incorporated into thermal noise power $N_{o} / 2$. The target BER $P_{b}$ of a QAM signal can be related to the number of symbols in the modulation constellation $M$ according to [14] as.

$$
P_{b}=\frac{4}{b}\left(1-\frac{1}{\sqrt{M}}\right) Q\left(\sqrt{\frac{3}{M-1} \gamma_{b}}\right)
$$




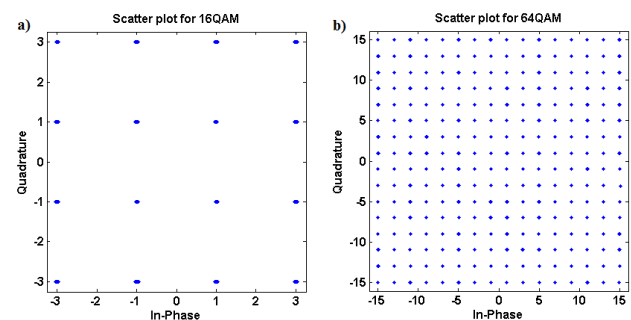

Fig. 3. a) Constellation diagram for 16QAM, b) Constellation diagram for 64QAM.

Here, BER is calculated over Rayleigh fading channel, and $\gamma_{b}$ is the average SNR per bit defined as $\gamma_{b}=E_{s} / N_{o}$. $E_{s}$ is the required energy per symbol at the receiver for a given BER requirement and $b$ is the constellation size. Using the Chernoff bound (3) can be reduced to:

$$
P_{b} \leq \frac{4}{b}\left(1-\frac{1}{\sqrt{M}}\right)\left(\frac{1.5 E_{s}}{N_{o}(M-1)}\right)^{-1} .
$$

Here $M=2^{b}$ and the symbol energy can be replaced by $\left(E_{b} b\right)$, where $E_{b}$ is the required energy per bit required at the receiver. Hence, from (4) we can obtain the average transmission energy per bit as follows:

$$
\frac{P_{b}}{4}\left(\frac{1}{1-\frac{1}{2^{\frac{b}{2}}}}\right) \leq\left(\frac{1.5 E_{b} b}{N_{o}\left(2^{b}-1\right)}\right)^{-1},
$$

When further simplified, we have:

$$
E_{b} \leq \frac{3}{2}\left(\frac{P_{b}}{4}\right)^{-1}\left(\frac{\sqrt{2^{b}}-1}{\sqrt{2^{b}}}\right)\left(\frac{N_{o}\left(2^{b}-1\right)}{b^{2}}\right),
$$

Finally we have:

$$
E_{b} \leq(1+\alpha) \frac{3}{2}\left(\frac{P_{b}}{4}\right)^{-1}\left(\frac{\left(2^{\frac{b}{2}}-1\right)\left(2^{b}-1\right)}{2^{\frac{b}{2}}}\right) \frac{N_{o}}{b^{2}} G d^{2} .
$$

Where $\alpha$ is the drain efficiency of the RF power amplifier and $d$ is the distance between the transmitter and the receiver $G$ is the antenna gain at both the transmitter and the receiver. We assume that path loss obeys the square root law. Now for the same transmitters, using same transmission energy but different modulation schemes (16QAM and 64QAM) (5) can be reduced to:

$$
\frac{\left(\sqrt{2^{16}}-1\right)\left(2^{16}-1\right)\left(d_{16}\right)^{2}}{\sqrt{\left(2^{16}\right) 16^{2}}}=\frac{\left(\sqrt{2^{64}}-1\right)\left(2^{64}-1\right)\left(d_{64}\right)^{2}}{\sqrt{\left(2^{64}\right) 64^{2}}},
$$

Here $d_{16}$ represents the distance covered by a QAM signal working on a constellation size of 16 and likewise $d_{64}$ represents the distance coverage of a signal transmitted using a QAM signal with a constellation size of 64 . Now if we simplify the above expression to obtain a relation between the distance covered by a $16 Q A M$ signal and a $64 Q A M$ signal
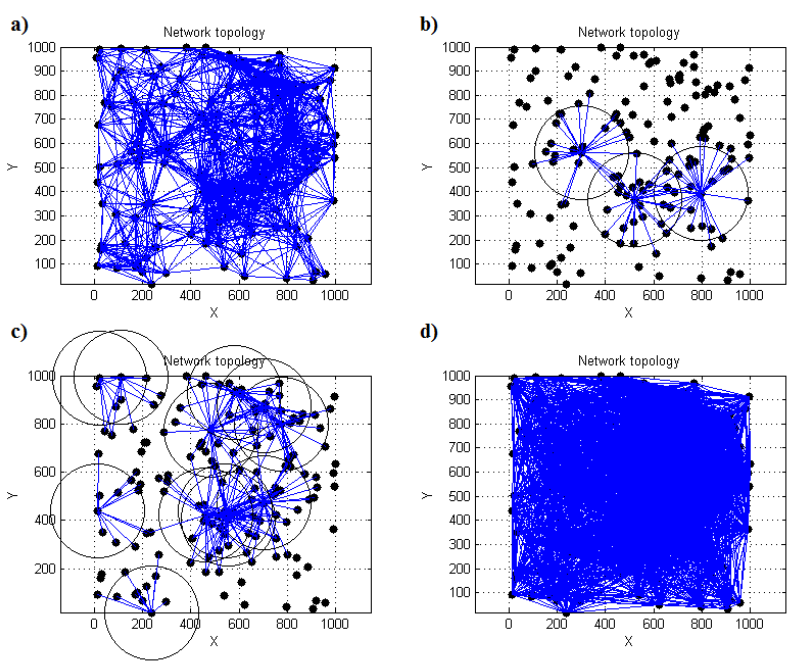

d)

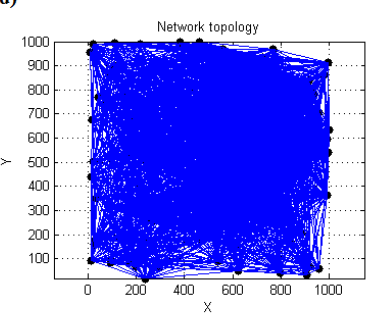

Fig. 4. Demonstration of topology modification mechanism. (a) Initial network with random link formation between nodes. (b) $3 \%$ nodes form long distance links using VRAM. (c) $10 \%$ nodes form long distance links using VRAM. (d) Network with $10 \%$ nodes forming links using VRAM and $90 \%$ nodes using random link formation.

using similar transmitters and under similar environmental conditions, we have:

$$
d_{16}=\frac{2 \sqrt{105}}{15} d_{64}
$$

$\mathrm{Eq}(6)$ denotes that by using the same amount of transmission energy and under the same BER requirements, a $16 Q A M$ signal travels $\frac{2 \sqrt{105}}{15}$ more distance when compared to $64 Q A M$. The main reason for covering a longer distance is the Euclidean distance between the constellation points of a $16 Q A M$ signal when compared to $64 Q A M$ as shown in Fig 3. A signal that has a greater distance in its constellation points is less prone to error. A noise signal has to have greater amplitude, in order to mix one constellation point into another at the receiver, for detection. Hence a $16 Q A M$ signal can travel a longer distance.

Using this concept, we have equipped nodes having a higher betweenness centrality, a lower order modulation scheme, to cover longer distances. Fig 4 shows the change in network topology after use of VRAM for an area of $1000 \times 1000 \mathrm{~m}^{2}$. Fig $4 \mathrm{a}$, shows the initial network setup where random links are formed between nodes, Fig $4 \mathrm{~b}$ and Fig $4 \mathrm{c}$ shows the area covered by nodes when $3 \%$ and $10 \%$ of nodes using VRAM where nodes form links with every neighboring node inside a specific region resulting in increased node degree. The final network formation is shown in Fig 4d where only $10 \%$ of nodes use VRAM for long range links and $90 \%$ nodes have random links of the initial setup.

\section{Simulation Results}

We evaluated our proposed model for a network consisting of omni-directional antennas, distributed randomly over a rectangular region. We study the impact of using VRAM for forming long distance relay paths. The simulations were run 
in Matlab. We compare our proposed model with methods proposed by [1] and [7] and name them Directional-WFB and Socially Inspired respectively. We also compare our results with a random network, where all the edges are randomly connected to analyze the change in node degree and name it Omni-directional. The results shown were averaged for all node pairs and 50 different topologies. We assume that the network can be disconnected due to limitation in transmission range and any node that cannot be accessed has a path length of infinity (we assume that 100000 is our infinity for better realization of APL).

For the first set of simulations, we vary the number of nodes in the network for a fixed network area of $1000 x 1000 \mathrm{~m}^{2}$. The percentage of nodes forming long distance relay paths is $10 \%$ for optimum performance [1][7]. Nodes are equipped with homogeneous amount of energy and use CSMA/CA for packet collision avoidance. It is assumed that the transmission radius of an omni-directional antenna is $250 \mathrm{~m}$. The distance covered by directional antennas is obtained from [1], stated as:

$$
r(\Theta)=r \sqrt{\frac{2 \pi}{\Theta}} .
$$

Where $r(\Theta)$ is the beam-length for a beam-width $\Theta$ and $r$ is the omni-directional transmission distance.

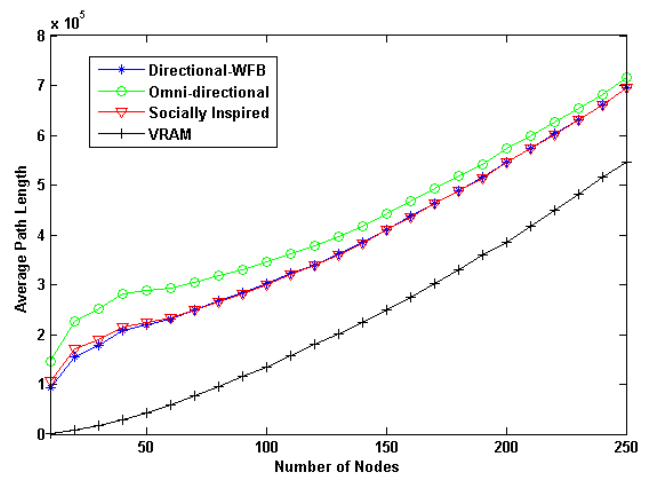

Fig. 5. Average path length with varrying number of nodes

Fig 5 denotes the variation in APL with change in the amount of nodes present in the network, under fixed network area. We vary the number of nodes in the range $[10-250]$ and observe that, Directional-WFB and Socially Inspired have a similar pattern, this shows that the distributed selection mechanism of node with highest degree has similar performance to the scheme provided by Socially Inspired, where a node is selected using an iterative mechanism for long distance relay path formation. VRAM out performs the rest due to its covered angle, the use of a lower order modulation scheme along with an Omni-directional antenna allows the node to have long distance communication covering $2 \pi$ radians. The use of directional antennas, as in Directional-WFB and Socially Inspired limit the node to communicate in a particular direction only.

As APL is the average distance of a particular node to all the nodes present in the network, so in case of a directional antenna, a node present in the exact opposite direction of the directional antenna's face will have a longer path length and a packet has to be relayed through neighboring nodes, this results in an increased path length. The major improvement in APL is due to the consideration of enhanced bridge crossing as shown in Fig 2. APL is measured in units of $10^{5}$ due to the consideration of random networks, where networks can be disconnected, resulting in high APL as nodes in a network can be in-accessible due to transmission range limitation. $V R A M$ is the only technique that caters for Enhanced Bridge Crossing, resulting in major improvement in APL. With a lower number of nodes, APL for Directional-WFB, Socially Inspired and Omni-directional increase due to widely spread nodes, forming network chunks. With the increase in number of nodes, these network chunks spread over and form a single network resulting in a smooth increase in APL.

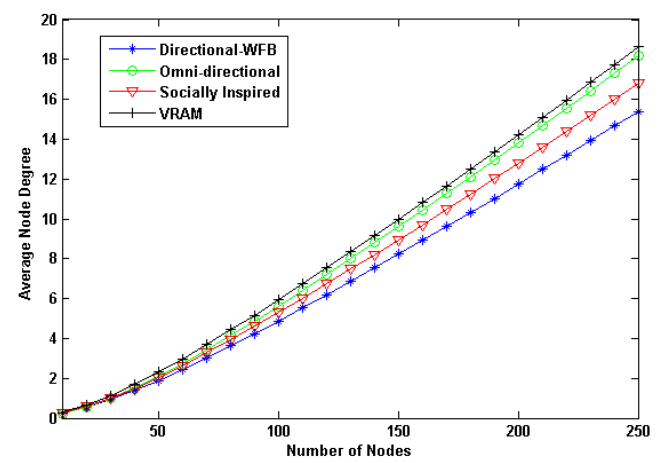

Fig. 6. Average node degree with varrying number of nodes.

Fig 6 represents the variation in Average Node Degree with the change in number of nodes for the same network area. Directional-WFB and Socially Inspired have a lower average node degree as they use directional antennas. Directional antennas form long distance relay path by breaking their current links and concentrating all the antenna power into one particular direction, this is executed at the loss of current links formed in all $2 \pi$ radians. On the other hand VRAM forms long distance Omni-directional links. It makes new long distance relay path, while maintaining its current communication paths, resulting in a higher average node degree.
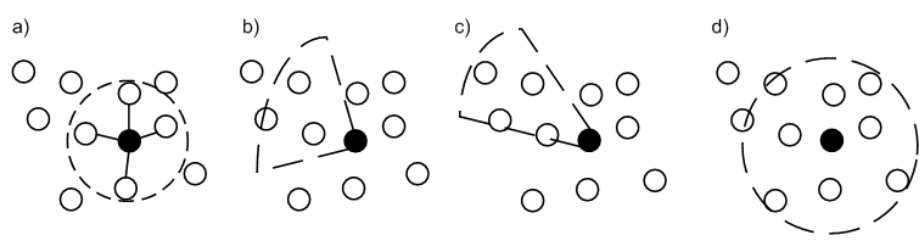

Fig. 7. Effect of changing beam width on beam angle and comparison node degree of VRAM.

Fig 7a shows the node degree of a node having links while using an Omni-directional antenna, as the beam-angle of a directional antenna is reduced the beam-length increases, decreasing the covered area and hence reducing the node degree as shown in Fig $7 \mathrm{~b}$ and Fig 7c. This trend results in reduced node degree of Directional-WFB and Socially Inspired. A relatively high node degree value represents that only $10 \%$ of nodes are equipped with directional antennas, The rest form links as shown in Fig 7a. VRAM follows a trend 
shown in Fig 7d, due to which $10 \%$ of nodes have higher node degree, resulting in an overall higher node degree. This enables nodes to relay data in any direction, reducing the overall cost of relaying messages.

For the second set of simulations, we vary the initial transmission radius in the interval $[100 m, 500 m]$. Number of nodes used is kept constant at 100nodes. The effect of varying the initial transmission radius on the APL and node degree is monitored.

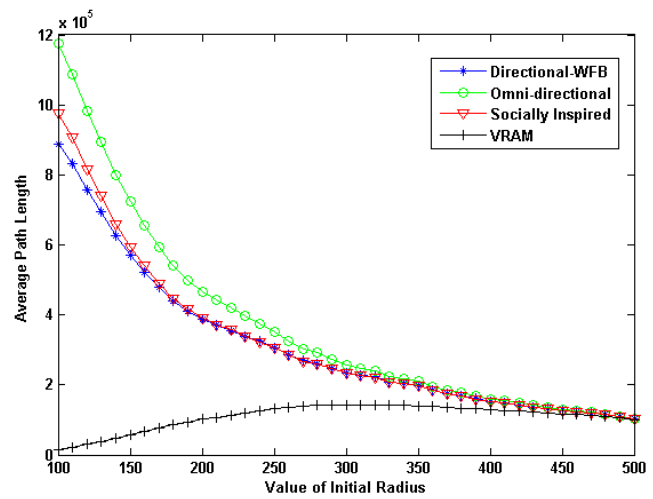

Fig. 8. Average path length with varying initial radius.

Fig 8 shows that as the initial radius of a node is increased APL tends to decrease for Directional-WFB and Socially Inspired, as they can relay messages to a greater distance with increase in initial radius. APL for Omni-directional decreases as more nodes are directly connected to a single node due to extended transmission radius. Directional-WFB, Socially Inspired and Omni-directional do not cater for the enhanced bridge crossing problem and hence have cases when the network is partitioned. As the value of the initial transmission radius increases, these inaccessible nodes become reachable and hence the APL decreases. Our mechanism of choosing the node with the highest betweenness centrality considers such nodes and hence the APL is always lower. The increase in APL is due to the increase in value of initial radius, more nodes come in direct contact, reducing number of hops and hence slightly increasing the APL. As the value of initial radius increases, the transmission radius of a node increases and more and more nodes can be accessed directly, reducing the APL at very high values of initial radius.

Fig 9 represents the variation in Average Node degree with varying the value of the initial radius. Similar to results shown in Fig 6, average node degree of VRAM is higher than that of simple Omni-directional antennas because in this mechanism, a node maintains its entire current links while building new long distance communication links.

\section{CONCLusion}

In this work, we have introduced the use of VRAM for formation of long distance communication links to reduce the APL of a WSN. Our proposed method overcomes the deficiencies faced by traditionally used directional antennas of limiting the beam-width to increase the beam-length. We also propose a mechanism that enables nodes to calculate the betweenness centrality measure by walking through a

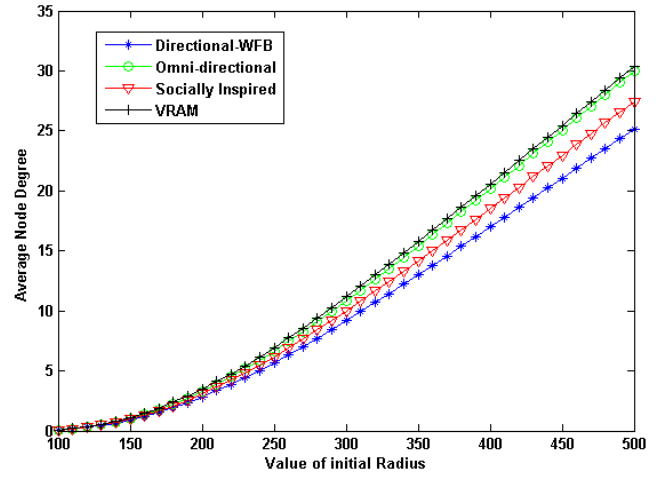

Fig. 9. Average node degree with varrying initial radius value.

lower number of nodes, using neighbor avoiding walk. We show using simulation results that significant performance is achieved by using variable modulation technique, over use of directional antennas for the formation of long distance relay paths. In future we intend to analyze the effect on the security of a network after adding these long distance paths.

\section{REFERENCES}

[1] A. Banerjee, R. Agarwal, V. Gauthier, C. Yeo, H. Afifi, and F. Lee, "A self-organization framework for wireless ad hoc networks as small worlds," Vehicular Technology, IEEE Transactions on, vol. 61, no. 6, pp. 2659-2673, 2012.

[2] S. Milgram, "The small world problem," Psychology today, vol. 2, no. 1, pp. 60-67, 1967.

[3] D. Watts and S. Strogatz, "The small world problem," Collective Dynamics of Small-World Networks, vol. 393, pp. 440-442, 1998.

[4] Z. Zhang, L. Rong, and C. Guo, "A deterministic small-world network created by edge iterations," Physica A: Statistical Mechanics and its Applications, vol. 363, no. 2, pp. 567-572, 2006.

[5] G. Sharma and R. Mazumdar, "Hybrid sensor networks: a small world," in Proceedings of the 6th ACM international symposium on Mobile ad hoc networking and computing, pp. 366-377, ACM, 2005.

[6] C. Verma, B. Tamma, B. Manoj, and R. Rao, "A realistic small-world model for wireless mesh networks," Communications Letters, IEEE, vol. 15, no. 4, pp. 455-457, 2011.

[7] E. Stai, V. Karyotis, and S. Papavassiliou, "Socially-inspired topology improvements in wireless multi-hop networks," in Communications Workshops (ICC), 2010 IEEE International Conference on, pp. 1-6, IEEE, 2010.

[8] P. Wang and I. Akyildiz, "Spatial correlation and mobility-aware traffic modeling for wireless sensor networks," IEEE/ACM Transactions on Networking (TON), vol. 19, no. 6, pp. 1860-1873, 2011.

[9] Z. Shelby, C. Pomalaza-Raez, H. Karvonen, and J. Haapola, "Energy optimization in multihop wireless embedded and sensor networks," International journal of wireless information networks, vol. 12, no. 1, pp. 11-21, 2005.

[10] M. Soltan, I. Hwang, and M. Pedram, "Modulation-aware energy balancing in hierarchical wireless sensor networks," in Wireless Pervasive Computing, 2008. ISWPC 2008. 3rd International Symposium on, pp. 355-359, IEEE, 2008.

[11] U. Brandes, "A faster algorithm for betweenness centrality*," Journal of Mathematical Sociology, vol. 25, no. 2, pp. 163-177, 2001.

[12] B. Hughes, "Random walks and random environments: Volume 2: Random environments (vol 2)," 1996.

[13] W. Webb and R. Steele, "Variable rate qam for mobile radio," Соттиnications, IEEE Transactions on, vol. 43, no. 7, pp. 2223-2230, 1995.

[14] S. Cui, A. Goldsmith, and A. Bahai, "Energy-efficiency of mimo and cooperative mimo techniques in sensor networks," Selected Areas in Communications, IEEE Journal on, vol. 22, no. 6, pp. 1089-1098, 2004. 